\title{
Evaluation of tribological properties of components of polymer composite self- lubricating materials
}

\author{
Kirill N. Dolgopolov ${ }^{1, *}$, and Dmitry N. Lyubimov ${ }^{2}$ \\ ${ }^{1}$ Rostov State Transport University (RSTU), 344038 Rostov-on-Don, Rostovskogo Strelkovogo Polka \\ Narodnogo Opolcheniya sq. 2, Russia \\ ${ }^{2}$ Branch of the Southern Federal University, 353461 Gelendzhik str.10-a Zastavnaya, Russia
}

\begin{abstract}
The paper presents data on the anti-wear properties of the basic components of polymer self-lubricating composite materials for heavy loaded friction units. In friction these components are released from the composite structure as a result of destructive processes or physical desorption with increasing temperature and involved in the metal plating of friction parts. Besides, it shows the wear form of a sample friction pair in comparison with I-40 base oil, often applied as a composite plasticizer, on the example of synergistic interference of unhardened epoxy-diane resin with highly dispersed mineral fillers of the serpentine group. The conducted study reveals the ability of minerals to reduce the wear rate of a sample friction pair as a result of the transferred film formation. The kinetic features of model samples wear under special conditions for testing ceramic materials are determined as well. In addition, the paper identifies the practicability to exploit layered silicates in the structure of polymer matrices as lubricating agents of heavy loaded friction units.
\end{abstract}

\section{Introduction}

At the present stage of machine construction and design the key purpose of the developed lubricating agents is to ensure the reliability of friction pairs while reducing the lubricant consumption and decreasing the cost of its production technologies. An important direction of work to maintain stable operation modes for heavy loaded metal friction pairs in subcritical conditions as applied to the «wheel-rail» unit of the railway transport is the development of solid lubricating coatings and methods of their formation on the protected surfaces directly during frictional interaction. The system like that works most effectively in the case of self-lubricating polymer composite materials, as a means of delivering lubricant to a strictly specified surface point, reducing its consumption. The introduction of additives to the bulk of the polymer matrix that improve the tribotechnical parameters of the initial material still remains the most effective way to solve the above mentioned problem given that improving the operational properties of polymer composites is the most crucial purpose of modern tribology [1-4].

\footnotetext{
* Corresponding author: kir-tribotex@yandex.ru
} 
It is necessary to possess experimental data on the effect of the polymer matrix base components on the friction bodies wear to obtain a synergistic effect while constructing polymer lubricators. The following components can be used as high-tech, non-deficient materials for polymer lubricants, according to the data provided in [5]:

- epoxy-diane resin, which has such prime technological advantages as low moisture permeability in the hardened form, high resistance to abrasive wear, and simplicity of preparing model samples;

- mineral filler of the serpentine group with lizardite predominance, characterized by high antiscuffing properties and tendency to form protective coatings [6-9].

The above listed components being mechanically mixed with the introduction of hardener, plastic grease based on I-40 oil (solid oil G) and mixture hardening for 15-24 are used to produce composite polymers. Perfect tribological properties of composites manufactured by the oilynite type with the usage of the above specified materials are considered in [10], however, the separate contribution of each oilynite component to ensure the tribological properties of the samples was not the subject of the investigation.

When composite self-lubricating polymer materials, produced by the oilynite type reach a certain threshold temperature or load value, determined by the technological aspects of their producing, the components of the lubricating medium start to escape («weep») from the bulk of the polymer matrix. This process occurs with the softening of the polymer chain that along with the classical friction transfer causes an additional self-lubricating effect.

\section{Purpose of the work}

To study tribological properties of the basic components of «oilynite» type polymers, prepared on the basis of a epoxy-diane resin filled with a highly dispersed mineral, as the most technological and non-deficit material.

\section{Methodology}

The tribological tests were conducted according to the «sphere on the disk» test scheme, presented in Fig. 1. The friction body is a disc made of structural alloyed steel $18 \mathrm{HG}$ (Russian State Standard 4543), polished to $\mathrm{Ra}=0.01 \mu \mathrm{m}$ roughness. Ceramic spheres of $6.35 \mathrm{~mm}$ diameter made of silicon nitride ( $\mathrm{Si} 3 \mathrm{~N} 4)$ are used as a counterbody. The tests were carried out applying the main provisions of DIN EN1071-13: 2010 «Special Technical Ceramics. Methods for Testing Ceramic Coatings. Part 13. Definition of the Wear Rate by the Part on the Disk Method» manual given that the investigations done in [6-10] established the ability of silicate fillers to form coatings whose properties are close to ceramic ones. During test there was applied $10 \mathrm{H}$ load; linear speed was equal to $4 \mathrm{~mm} / \mathrm{sec}$.

In the course of the experiment we measured the values of the body's coefficient of friction. Furthermore, we studied the topology of surface damages while using each type of lubricant (a sample of a lubricant composition).

The selected test scheme allows minimizing the influence of the viscosity of the reference medium, and the contact geometry provides: a) fast run-in, b) absence of edge effects; c) instrumental simplicity of the friction track analysis; d) good material adhesion; e) high pressures and thermal tension of the friction unit, which is typical for heavy loaded friction pairs. 


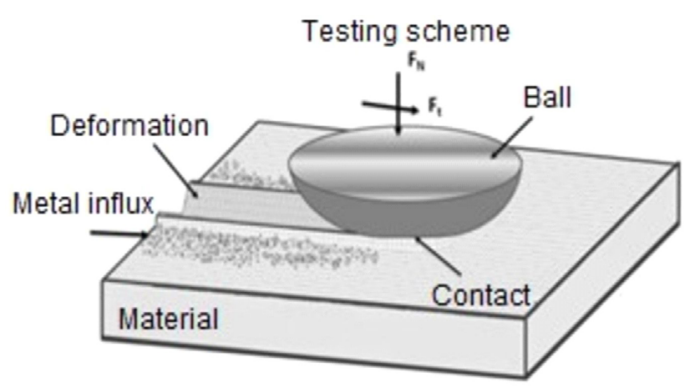

a)

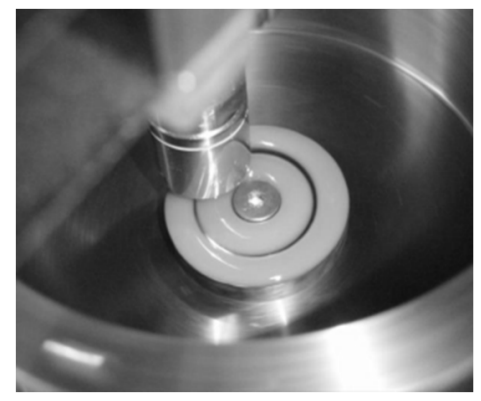

b)

Fig. 1. Scheme of materials interaction during tests: a) basic diagram of the friction unit; b) an image of a friction pair.

Mineral filler powder was ground on a laboratory bead mill MiniCer (Netzch) to not exceeding $1 \mu \mathrm{m}$ size. The nature of the phase composition of the dispersed material was identified on a DRON-3 X-ray diffractometer (a cobalt anode source operating at $20 \mathrm{kV}$ ). $3 \%$ wt. microdispersed filler was introduced with intensive stirring using IKA T25 disperser homogenizer to unhardened ED-20 epoxy-diane resin, pre-heated to a thin fluid state $\left(60^{\circ} \mathrm{C}\right)$.

At the end of each tribological test cycle, we determined the wear value and the characteristics of the worn-out surface topography using a probe profilometer. The analytical complex is equipped with a light-optical microscope $(25 \ldots 1000$ times magnification), which makes it possible to observe structures on the friction surface in reflected light.

The following materials were exploited as sample test media:

Lubricant № 1 - pure ED-20 epoxy resin (Russian State Standard 10587);

Lubricant № 2 - industrial oil I-40 (Russian State Standard 20799);

Lubricant № 3 - ED-20 epoxy resin, containing 3\% wt. serpentine powder.

The use of I-40 oil as a reference sample is conditioned by its wide spread in the composition of grease self-lubricating composite materials, in which the given oil is thickened with various types of thickeners (Solid oil, Lithol-24). Heating or intensive mechanical disturbance, occurring in the friction unit during its long operation, leads to the oil release from the grease composition. The given oil acts as lubricating and plasticizing medium in the friction of self-lubricating composites.

In accordance with the above mentioned facts it is of great interest to study the frictional behaviour of the separate components of self-lubricating composites to improve the procedures and technologies of their production.

\section{Results and discussion}

The coefficient of friction values of lubricating composition samples are presented in Table 1.

Table 1. Lubricating compositions test results.

\begin{tabular}{|c|c|c|}
\hline № & Sample & Coefficient of friction \\
\hline 1 & Lubricant №1 & 0.0867 \\
\hline 2 & Lubricant №2 & 0.0806 \\
\hline 3 & Lubricant №3 & 0.1201 \\
\hline
\end{tabular}

The dynamics of the coefficient of friction changes is demonstrated in Fig. 2. 


\section{Coefficient of friction}

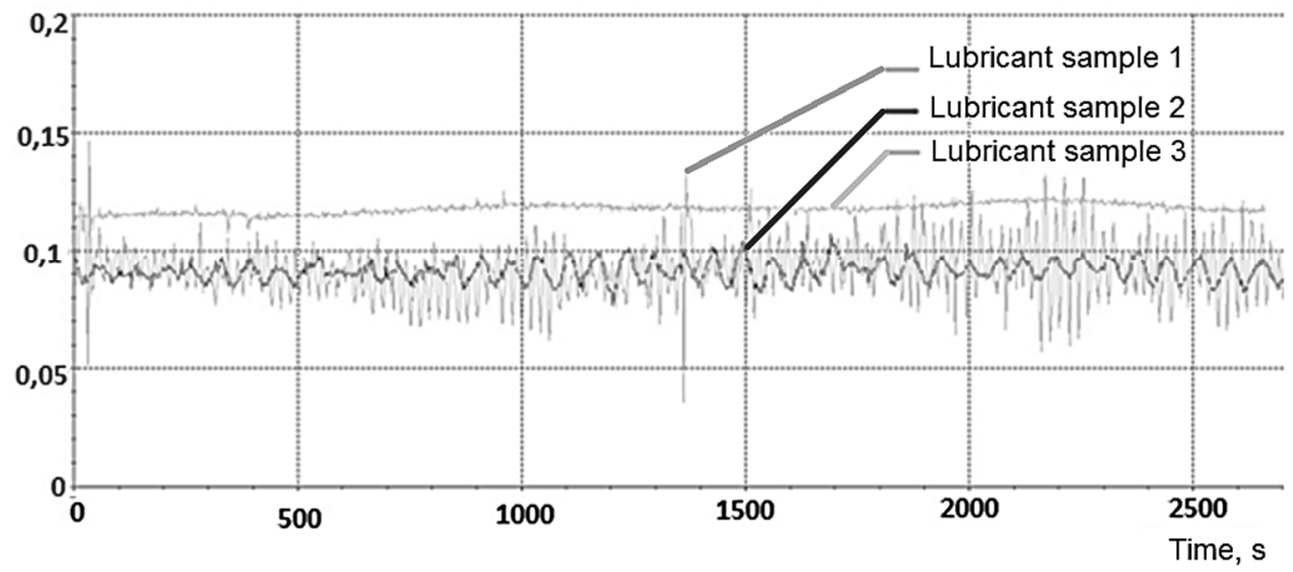

Fig. 2. Graph of the coefficient of friction changes (upper tribogram is sample № 3).

It was established that the use of mineral-filled epoxy resin contributes a more stable operation of the friction unit to the applied test scheme. It is accompanied by smaller fluctuations in the coefficient of friction, indicating the destruction of the lubricating film. The given fact cannot be explained by the difference in viscosity of the exploited lubricating media, since samples № 1 and № 3 prepared by the same type of lubricating medium manifest diverse frictional behavior.

Particular attention is drawn to the fact that, despite the great stability, the coefficient of friction in case of applying sample № 3 has a higher value. It proves that the mineral filler is capable to form a tread film separating the friction parts, and not chaotically distributed in the contact zone, but the film material itself cannot be considered as an anti-friction layer. The formation of the protective layer is indicated by the profilogramm of the disc friction body surface and the microphotography of the sphere surface, presented in Fig. 3 and Fig. 4.

Fig. 3 shows the profilograms of the friction surface rubbed during the testing of various types of lubricants

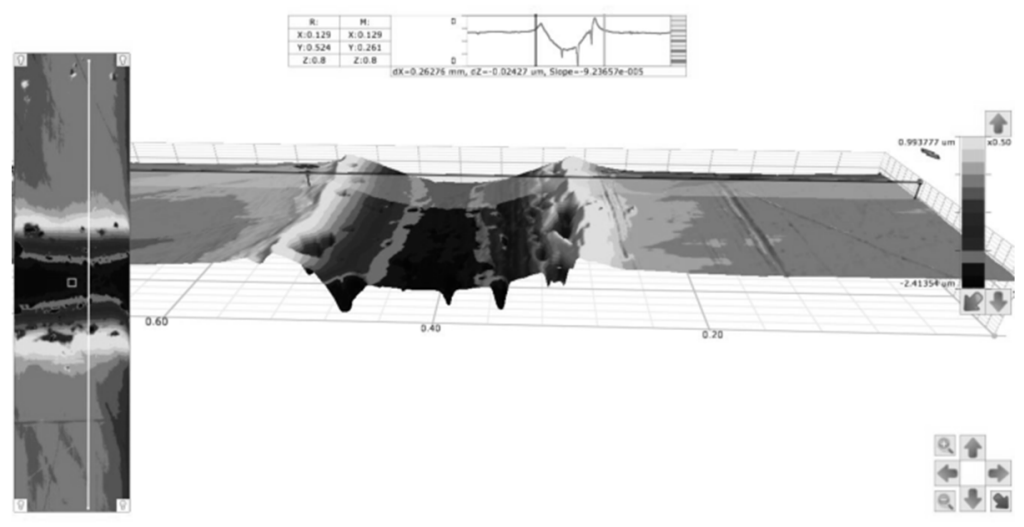

a) 


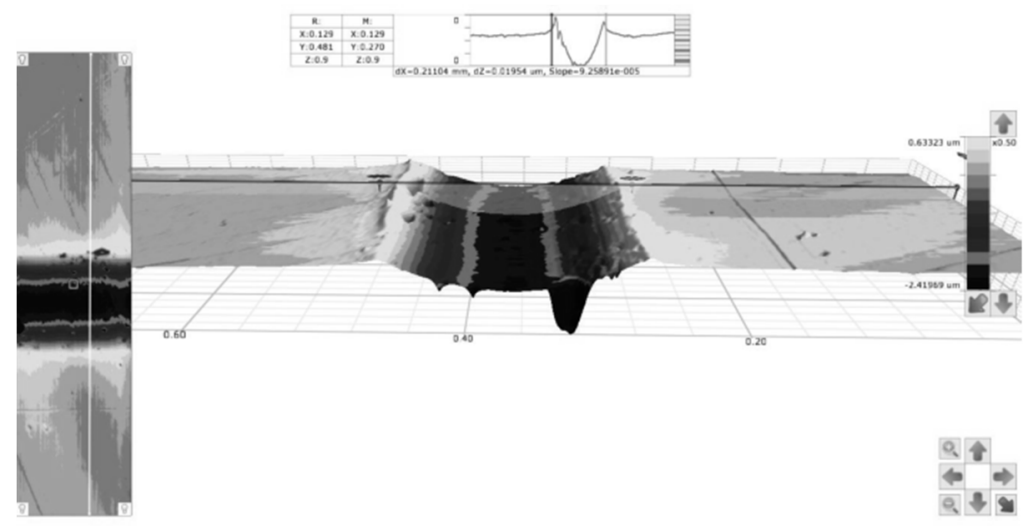

b)

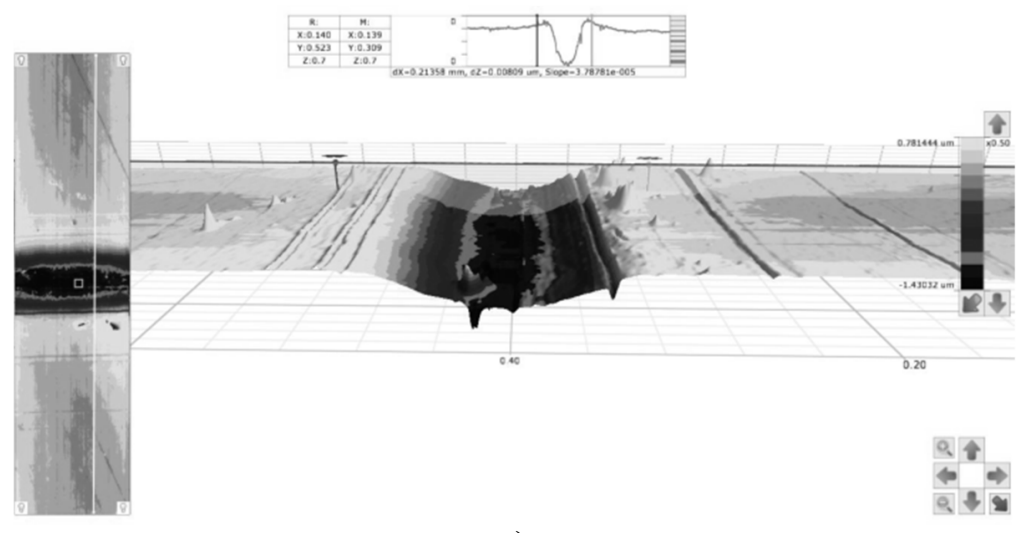

c)

Fig. 3. Profilogram of the friction surface while using lubricators: a) lubricant № 1, b) lubricant № 2, c) lubricant № 3 .

Fig. 3 clearly demonstrates the formation of scuffing with a large depth damage of the matrix metal bulk, when using lubricator № 1 and № 2. At the same time it represents the absence of similar damages while using mineral filler in the lubricant composition № 3. Attention is drawn to the narrower damage area of the friction body in the case of lubricant № 3. It is indirectly evidence the formation of a protective film on the friction body surface, since its destruction occurs mainly in the area of maximum temperatures and mechanical stresses, i.e. at the upper point of the spherical indenter. A more uniform colour range in Fig. 3c, exemplifying the depth of the recess of the metal friction body in wear, confirms the homogeneity of the protective film formed on its surface. The conducted analysis of the colour filling of the wear indentation edges rubbed on the friction body indirectly indicates that when using lubricants № 1 and № 2, the metal damage was accompanied by tears, confirming the generation of high local temperatures in the friction area and metal melting. While using the lubricator № 3, the friction body damage occurs due to the plastic penetration of the indenter without melting the metal: the edges of the wear indentation are smooth with an insignificant built-up of extruded material along the periphery. Fig. 4 displays micro images of the worn-out surface of a ceramic spherical indenter. 


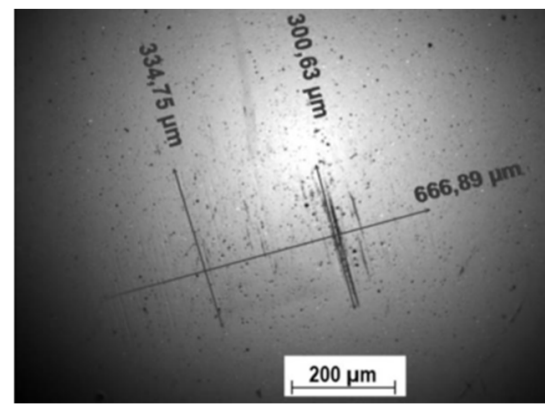

a)

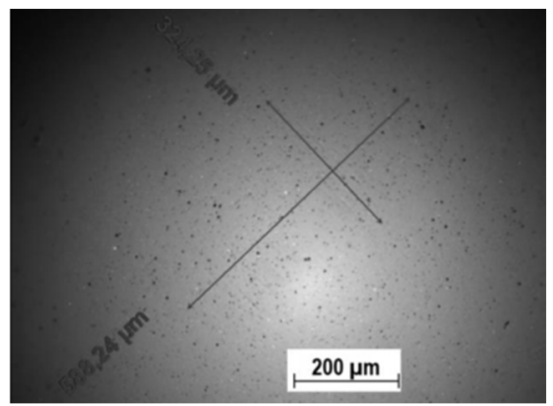

b)

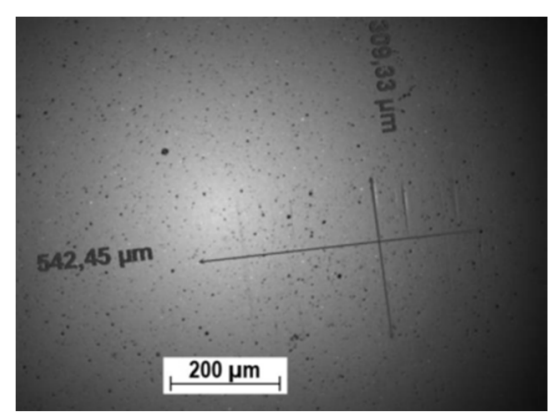

c)

Fig. 4. Micro images of the worn-out surface of a ceramic counterbody: a) lubricant № 1, b) lubricant № 2, c) lubricant № 3 .

The surface microscopy of the spherical indenter confirms the previously revealed regularities of wear during the formation of protective films on the friction surface when localizing the damage areas of the friction pair materials (the indenter operating in № 3 lubricant medium has the smallest linear dimensions of the worn-out section). Besides, it is possible to notice the formation of scuffing when using lubricants № 2 and № 3. In addition, while applying lubricant № 3, the surface is characterized by a high purity, which indicates for the latter that there are no local seizure areas and the predominance of a prolonged form of fatigue wear in the form of gradual accumulation of surface defects with subsequent spalling of the ceramic sample surface layer clearly visible in Fig. 4, b and 4, c. The results of studying the ceramic indenter wear perfectly correlate with the data presented in Table 1 on wear of the friction body: the basic medium № 3 is characterized with the smallest coefficient of friction.

\section{Conclusions}

The carried out research allows concluding the following. It was established that the introduction of a layered silicate in the form of a highly dispersed serpentine powder can be considered as a wear inhibitor, allotting wear-resistant and antiscuffing properties to a lubricating medium that does not have specified tribological functions. The discovered protective properties are enhanced as the contact time of the mineral particles with the friction surface increases. It manifests the process of forming an abrasion-resistant film separating metal surfaces. That is the reason why the exploitation of layered silicates in the polymer matrix structure to lubricate heavy loaded friction units is a promising technology 
for increasing their reliability, especially taking into account their low cost and nondeficiency.

The study was carried out at the expense of the Russian Science Foundation grant (project No. 14-2900116) by the project participant Dolgopolov K.N. in Rostov State Transport University.

\section{References}

1. P. V. Sysoev, M. M. Bliznets, A. K. Pogosyan, AntiFriction Epoxy Composites in Machine-Tool Construction (Navuka i technika, Minsk, 1990)

2. A. K. Pogosyan, K. V. Oganesyan, A. R. Isadzhanyan, Journal of Friction and Wear, 23 (3), 324 (2002)

3. A. S. Kuzharov, N. Yu. Onishchuk, Metall Plating Lubricants/ Durability of Machine Friction Parts: Proceedings of Scientific Researches, 3 (Mechanical Engineering, Moscow, 1988)

4. O. E. Shkurakova, The Development of Compositions, the Study of the Structure and Properties of Anti-Friction Composites with Additives of Modified Lignin. Abstract of Candidate of Engineering Sciences Thesis (Novocherkassk, 2010)

5. O.V. Zazimko, I.F. Pustovoy, D.N. Lubimov, K.N. Dolgopolov, Patent WO2010021569 (A1); Priority number(s): RU20080133853 20080819; 25.02.2010.

6. K.N. Dolgopolov, D.N. Lyubimov, A.T. Kozakov, A.V. Nikolskiy, E.A. Glazunova, Journal of Friction and Wear, 35, 2, 188 (2014)

7. K.N. Dolgopolov, V.L. Potekha, D.N. Lyubimov, Tri-Bology of Geomodified Lubricants: Monograph (Grodno State Agrarian University, Grodno, 2013)

8. K.N. Dolgopolov, D.N. Lyubimov, I.V. Kolesnikov, Physics and Chemistry of Glass, 42, 3, 420 (2016)

9. K.N. Dolgopolov, D.N. Lyubimov, A.T. Kozakov, A.V. Nikolskiy, E.A. Glazunova, Journal of Friction and Wear, 33, 2, 90 (2012)

10. K.N. Dolgopolov, I.V. Kolesnikov, E.L. Melnikov, Repair, Restoration, Modernization, 4, 23 (2018) 